\title{
Overexpression and delocalization of claudin-3 protein in MCF-7 and MDA-MB-415 breast cancer cell lines
}

\author{
MARIA C. TODD ${ }^{1}$, HEATHER M. PETTY ${ }^{1}$, JONATHAN M. KING ${ }^{2}$, \\ BRYTANIE N. PIANA MARSHALL ${ }^{1}$, REBECCA A. SHELLER ${ }^{1}$ and MARIA E. CUEVAS ${ }^{1}$ \\ ${ }^{1}$ Biology Department, Southwestern University, Georgetown, TX 78626; \\ ${ }^{2}$ Biology Department, Trinity University, San Antonio, TX 78212, USA
}

Received June 11, 2014; Accepted January 29, 2015

DOI: $10.3892 / \mathrm{ol} .2015 .3160$

\begin{abstract}
Tumor-specific deregulated expression of claudins, integral membrane proteins found in tight junctions (TJs), has indicated a possible role for TJ disruption in cancer progression. The current study demonstrates the marked overexpression of claudin-3 protein in two breast cancer cell lines of metastatic origin (MCF-7 and MDA-MB-415). Immunofluorescence and differential detergent fractionation analyses revealed that, although claudin-3 was primarily localized at cell junctions, it was also detected intracellularly. Similarly, the siRNA-mediated suppression of claudin-3 did not considerably affect its pattern of subcellular distribution relative to mock-transfected cells. However, there appeared to be a preferential loss of claudin-3 signal in the cytoskeletal fraction. Wound-healing assays were conducted to assess the effect of endogenous overexpression versus siRNA-mediated suppression of claudin-3 on cellular motility in MCF-7 cells. Suppression of claudin-3 protein levels resulted in a marked decrease in the rate of cellular motility relative to mock-transfected cells. These findings suggest that overexpression of claudin-3 may be important in disrupting TJ integrity and thus contribute to enhanced cellular motility, a key component of tumor progression.
\end{abstract}

\section{Introduction}

The incidence of breast cancer continues to rise worldwide, particularly in the USA where approximately one in eight females will be diagnosed with breast cancer during her lifetime (1). While early stage breast cancer is readily treatable, patients with metastatic disease pose a greater therapeutic challenge and account for the majority of breast cancer-related mortalities. This is largely due to the paucity of knowledge

Correspondence to: Dr Maria C. Todd, Biology Department, Southwestern University, 1001 East University Avenue, Georgetown, TX 78626, USA

E-mail: toddm@southwestern.edu

Key words: cell motility, tight junctions, metastasis with regard to the underlying molecular mechanisms associated with malignant progression.

Regulated cellular proliferation and differentiation are dependent upon functional tight junctions (TJs), and the loss of TJ integrity may be important in cancer development and progression. Located immediately beneath the apical surface of adjacent endothelial and epithelial cells, TJs form an effective barrier to the diffusion of solutes through the paracellular pathway and exhibit ion-selective permeability in a cell type-dependent manner. In association with adherens junctions, TJs have been shown to establish and maintain epithelial cell polarity by preventing the diffusion of membrane proteins and lipids between the apical and basolateral regions of the plasma membrane. Recent studies also suggest that the TJ plaque proteins, located on the cytoplasmic side of the TJ, are important for the integration of signaling molecules that regulate processes including gene transcription, cellular proliferation, differentiation and morphogenesis. This was reviewed by Turksen and Troy (2).

Claudins, the predominant integral membrane proteins that form the backbone of TJs, are required for the assembly, barrier and pore functions of vertebrate TJs (2). Deregulated expression of various claudin proteins has been reported in breast cancer; overexpression of claudin-3 and claudin- 4 was demonstrated in 62 and $26 \%$ of primary breast tumors, respectively (3). Furthermore, Lanigan et al (4) showed claudin-4 expression in $90.9 \%$ of primary breast cancers, with the highest levels of claudin- 4 associated with high grade breast tumors. Notably, although immunohistochemical analysis indicated a decrease in claudin-4 in 64\% of grade I breast tumors, its expression was found to be robust in grade II and III tumors (5). In contrast to the general upregulation of claudins- 3 and -4 in breast cancer, two other claudin proteins have been shown to be downregulated in breast cancer. Specifically, Tokés et al (5) reported a decrease in claudin-1 protein expression in $80 \%$ of invasive ductal breast carcinomas. In addition, claudin-7 expression has been shown to decrease with increasing breast tumor grade $(6,7)$. These studies indicate that deregulated levels of a number of claudin proteins may contribute to breast tumorigenesis.

The present study aimed to investigate the potential role of claudin-3 in tumor progression, by assessing the levels of this protein in panels of normal tissues (to examine the 
basal expression range) and in metastatic breast cancer cell lines MCF-7 and MDA-MB-415 (adenocarcinomas derived from pleural effusions) and by evaluating the effects of siRNA-mediated suppression of claudin-3 on cellular motility.

\section{Materials and methods}

Cell lines. The following human breast cancer cell lines were obtained from the American Type Culture Collection (Manassas, VA, USA): MCF-7 (catalog no. HTB-22), MDA-MB-415 (catalog no. HTB-128) and MDA-MB-157 (catalog no. HTB-24). The three cell lines were cultured in minimum essential media (MEM; catalog no. 11095-080) supplemented with $10 \%$ fetal bovine serum (catalog no. 16140-071) and 1\% penicillin-streptomycin-L-glutamine (catalog no. 10378-016), all of which were obtained from Life Technologies (Grand Island, NY, USA). Human mammary epithelial cells (HMEC; catalog no. CC-2551) were obtained from Lonza (Walkersville, MD, USA) and cultured in mammary epithelial basal medium (catalog no. CC-3151; Lonza) supplemented with the MEGM Bullet Kit (catalog no. CC-3150; Lonza). All cells were maintained in a humidified atmosphere of $5 \% \mathrm{CO}_{2}$ at $37^{\circ} \mathrm{C}$ for $7-10$ days prior to use.

Western blot analysis. Log phase cells were harvested with trypsin-EDTA $(0.25 \% / 1 \mathrm{mM}$; Life Technologies) and subjected to centrifugation at $81.7 \mathrm{x}$ g for $5 \mathrm{~min}$. The cell pellets were then resuspended in $1 \mathrm{X}$ sample buffer $(10 \% \mathrm{v} / \mathrm{v}$ glycerol, $1 \%$ SDS, $0.125 \% \mathrm{w} / \mathrm{v}$ bromophenol blue and $0.04 \mathrm{M}$ Tris $\mathrm{pH} 7.0$ ) plus $10 \% \beta$-mercaptoethanol, and subjected to gel electrophoresis on precast $10 \%$ or $12 \%$ SDS-polyacrylamide gels (catalog no. 456-1033 and 456-1043; BioRad, Hercules, CA, USA). Following transfer to polyvinylidene fluoride membranes (catalog no. IPVH304F0; Merck Millipore Ltd, Co. Cork, Ireland), the membranes were incubated with the following antibodies in $5 \%$ milk/phosphate buffered saline (PBS) for $2 \mathrm{~h}$ at room temperature: $1 \mu \mathrm{g} / \mathrm{ml}$ Rabbit anti-claudin-1 (dilution, 1:1,000; catalog no.49339; Cell Signaling Technologies, Inc, Beverly, MA, USA); $2 \mu \mathrm{g} / \mathrm{ml}$ rabbit anti-claudin-3 (dilution, 1:250; catalog no. 34-1700; Life Technologies); $3 \mu \mathrm{g} / \mathrm{ml}$ mouse anti-claudin-4 (dilution, 1:167; catalog no. 32-9400; Life Technologies); and $1 \mu \mathrm{g} / \mathrm{ml}$ rabbit anti-actin (I-19; dilution, 1:200; catalog no. SC1616-R; Santa Cruz Biotechnology, Santa Cruz, CA, USA). The membranes were subsequently incubated in a 1:3,000 dilution of goat anti-rabbit (catalog no. 172-1019; BioRad, Hercules, CA, USA) or goat anti-mouse HRP-conjugated secondary antibodies (catalog no. 170-6516; BioRad) in $5 \%$ milk/PBS for $1 \mathrm{~h}$ at room temperature. Signals were visualized using Amersham ECL Prime Western Blotting Detection Reagent (catalog no. RPN2232; GE Healthcare, Buckinghamshire, UK).

Differential detergent cell fractionation. Using the ProteoExtract Protein Extraction Kit (catalog no. 539791; Calbiochem/EMD Chemicals, Inc, San Diego, CA, USA), $\sim 1 \times 10^{6} \log$ phase cells were incubated sequentially in four extraction buffers to yield cytosolic, membranous, nuclear and cytoskeletal fractions, following the manufacturer's instructions.

Transient siRNA transfection. Approximately $1 \times 10^{5}$ cells were plated into $100 \mathrm{~mm}$ wells and cultured in MEM supplemented with $10 \%$ fetal bovine serum and $1 \%$ penicillin-streptomycin-L-glutamine. After reaching $30 \%$ confluency, cells were transfected with 20 pmol Silencer Select siRNA targeted to claudin-3 (catalog no. s3444; Life Technologies) using $6 \mu \mathrm{l} /$ well DharmFect I cationic lipid (catalog no. T-2001; Fisher Scientific, Pittsburgh, PA, USA) in serum-free medium. For controls, cells were mock-transfected with serum-free medium. Following incubation at $37^{\circ} \mathrm{C}$ for $24 \mathrm{~h}$, the medium was replaced with fresh medium containing serum.

Indirect immunofluorescence analysis. Log phase cells were harvested, plated at a density of $5 \times 10^{5}$ cells per chambered cover slide (catalog no. 177437; LabTek, Fisher Scientific) and incubated at $37^{\circ} \mathrm{C}$ until $80 \%$ confluency was reached. Cells were rinsed three times with pre-cooled PBS and fixed in pre-cooled $95 \%$ ethanol for 30 min on ice. Following blocking with $1 \%$ bovine serum albumin, the cells were incubated with $1 \mathrm{mg} / \mathrm{ml}$ rabbit-anti claudin-3 overnight at $4^{\circ} \mathrm{C}$. Cells were subsequently counterstained with goat anti-rabbit-Alexa-488 (dilution, 1:400; catalog no. A-11034; Life Technologies) for $1 \mathrm{~h}$, rinsed with PBS and treated with Prolong Gold antiFade Reagent (catalog no. I37156; Life Technologies). Images were collected using a Nikon TE2000U eipfluorescent system with a PlanFluor 40X objective and a Coolsnap HQ camera.

Wound-healing assay. Claudin-3 siRNA-transfected and mock transfected control cells were plated at $2.5 \times 10^{5}$ cells per well into Cytoselect wound-healing assay plates (catalog no. CBA-120; Cell Biolabs San Diego, CA, USA) and grown until $95 \%$ confluent. Each well contained an insert that created a defined, rectangular wound field. Following removal of the inserts, the cells were stained (stain included in Cytoselect kit) and photographed on days $0,1,2$ and 3 to monitor migration of the cells into the wound field. Protein extracts were prepared from the mock- and siRNA-transfected cells (as described previously) on days 0 and 3 , and subjected to western blot analysis to assess the degree of claudin-3 suppression.

\section{Results}

Claudin-3 protein expression in normal human tissues. To determine the level and variation of expression of claudin-3 in normal tissues, a panel of protein extracts derived from human bladder, breast, cervix, kidney, ovary, placenta, prostate, testis and uterus were analyzed using a pre-made tissue western blot (ProSci). The $23 \mathrm{kDa}$ claudin-3 band was detectable in all tissues with the exception of the bladder, cervix, placenta and uterus (Fig. 1). The most intense signal was observed in the lane containing normal breast tissue.

Overexpression of claudin proteins in metastatic breast cancer cell lines. Immunoblot analysis was used to assess the expression of claudin-1, -3 and -4 proteins in three breast cancer cells lines (MCF-7, MDA-MB-415 and MDA-MB-157), all of which 


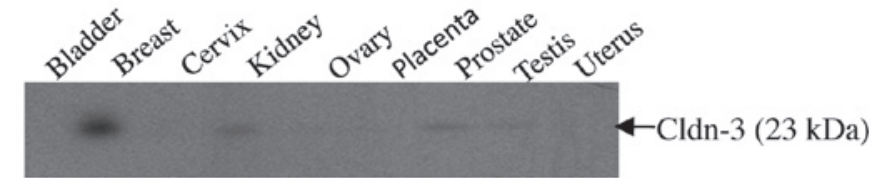

Figure 1. Claudin-3 protein expression in normal human tissues. A pre-made western blot (ProSci) containing a panel of protein extracts derived from different human tissues was probed with anti-claudin-3. The $23 \mathrm{kDa}$ claudin- 3 band was detected in all tissues with the exception of the bladder, cervix, placenta and uterus. The most intense signal was observed in the lane containing normal breast tissue. Cldn, claudin.
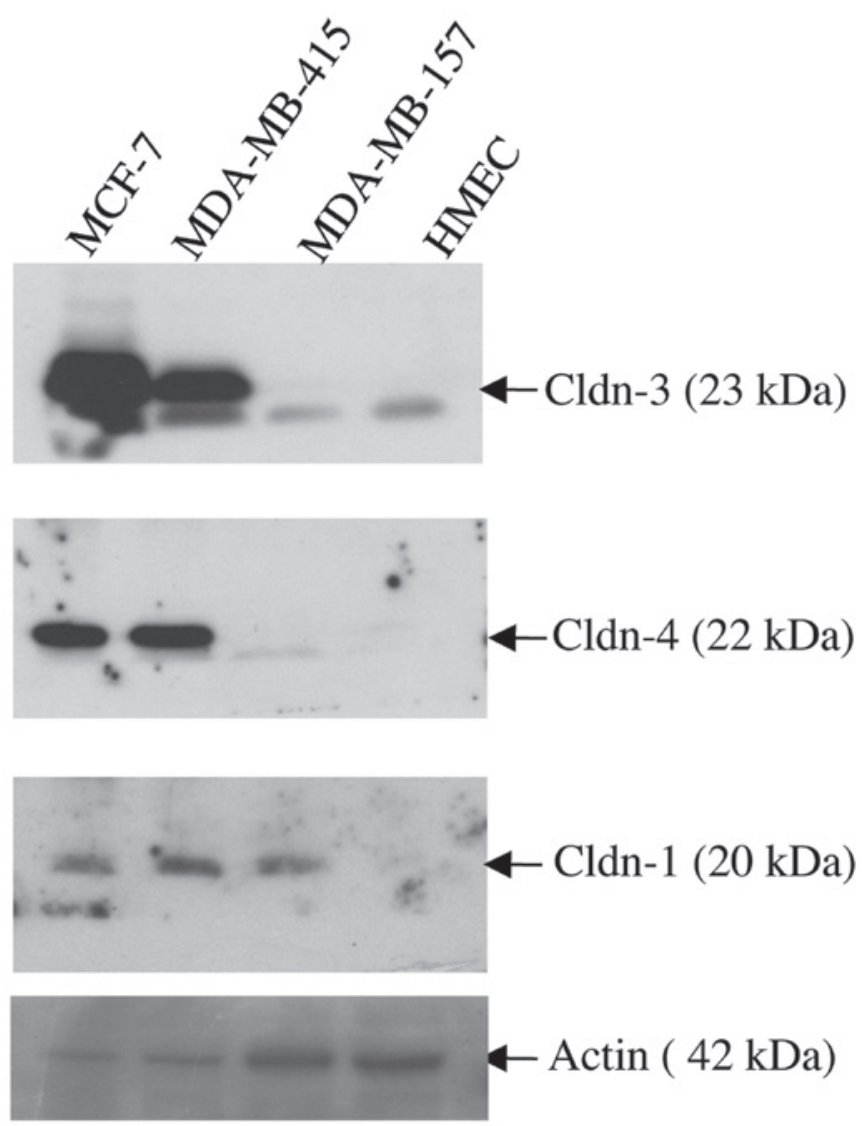

Figure 2. Overexpression of claudin proteins in the metastatic breast cancer cell lines MCF-7 and MDA-MB-415. Three breast cancer cell lines (MCF-7, MDA-MB-415 and MDA-MB-157) and the normal breast epithelial cell line, HMEC were subjected to immunoblot analysis using antibodies to claudin-1, -3 , and -4 . Overexpression of all three claudin proteins, particularly claudins- 3 and -4 , was observed in the cancer cell lines relative to the HMEC cells. HMEC, human mammary epithelial cells; cldn, claudin.

were derived from patients with metastatic disease (Fig. 2). As a control, the expression of the same three proteins was assessed in HMEC cells. Whereas the HMEC cells expressed barely detectable levels of each of the three claudin proteins, marked overexpression of claudins-3 and -4 was observed in the MCF-7 and MDA-MB-415 cell lines. By contrast, the MDA-MB-157 cell line expressed a similar level of expression of claudins-3 and -4 as the HMEC cells in the form of a lower molecular weight band $(\sim 23 \mathrm{kDa})$. All three cancer cell lines expressed readily detectable levels of claudin-1 protein relative to the virtually undetectable expression of claudin- 1 demonstrated by the HMEC cells.
Delocalization of overexpressed claudin-3 protein in the MCF-7 and MDA-MB-415 breast cancer cell lines. Indirect immunofluorescence and differential detergent cell fractionation were utilized to determine and compare the effect of endogenous overexpression versus siRNA-mediated suppression of claudin-3 protein on its sub-cellular localization in the two claudin-3 overexpressing breast epithelial cell lines, MCF-7 and MDA-MB-415.

In the immunofluorescence analysis, MCF-7 and MDA-MB-415 cells showed marked fluorescence at cell junctions, consistent with the established role of claudin-3 in TJs (Fig. 3A and B). In addition, evidence of intracellular claudin-3 staining was detected in the two cell lines. To determine whether or not the intracellular fluorescence was claudin-3-specific, signal intensities following siRNA-mediated suppression of claudin-3 in the MCF-7 cells were calculated following subtraction of secondary antibody controls. The cells were visualized by differential interference contrast (DIC) microscopy (Fig. 4, bottom panels) and claudin-3 localization was visualized by immunofluorescence (Fig. 4, top right panels). The immunofluorescence images were then superposed onto the DIC images (Fig. 4, top left panels), enabling the comparison of claudin-3 signal intensities, at the cell junctions and within the cells, in the mock- versus siRNA-transfected conditions. Successful transfection of claudin-3-specific siRNA resulted in the loss of claudin-3 signal at cell junctions, however, a small fraction of the cells showed junctional staining, which suggested a transfection efficiency of $<100 \%$ (Fig. 4B top right panel). Notably, a significant $(55 \%)$ reduction in intracellular fluorescence was observed in siRNA-transfected cells that showed a decrease in junctional fluorescence, and in those that demonstrated claudin-3 signal at cell junctions $(\mathrm{P}<0.001)$ (determined by analysis of variance with Tukey post-hoc comparison; Fig. 4B, top right panel). These data suggest that the intracellular signal observed in the breast cancer cell lines was claudin-3 specific and not the result of non-specific background staining.

For the differential detergent cell fractionation analysis, mock- and claudin-3 siRNA-transfected MCF-7 (Fig. 5A) and MDA-MB-415 (Fig. 5B) cells were incubated sequentially in four extraction buffers to yield cytosolic, membranous, nuclear, and cytoskeletal fractions. Immunoblot analysis of proteins from each fraction of the mock-transfected cells indicated that the majority of claudin-3 was localized in the membrane (Fig. 5). Lower levels of claudin-3 were also observed in the cytosolic, nuclear and cytoskeletal fractions. siRNA-mediated suppression of claudin-3 resulted in a marked reduction in the overall level of claudin-3 protein, but did not considerably affect its pattern of subcellular distribution relative to the mock-transfected cells; however a more noticeable decrease in signal was observed in the cytoskeletal fraction. In addition to the $23 \mathrm{kDa}$ claudin-3 protein, a lower molecular weight band was detected in the mock- and siRNA-transfected whole cell extracts and cytosolic fractions of the MCF-7 and, to a lesser extent, MDA-MB-415 cells, which may correspond to a processed form of the claudin-3 protein that is found in the cytosol (Fig. 5).

Effect of claudin-3 expression level on cell motility. As normal breast epithelial cells are characteristically non-motile, the 
A

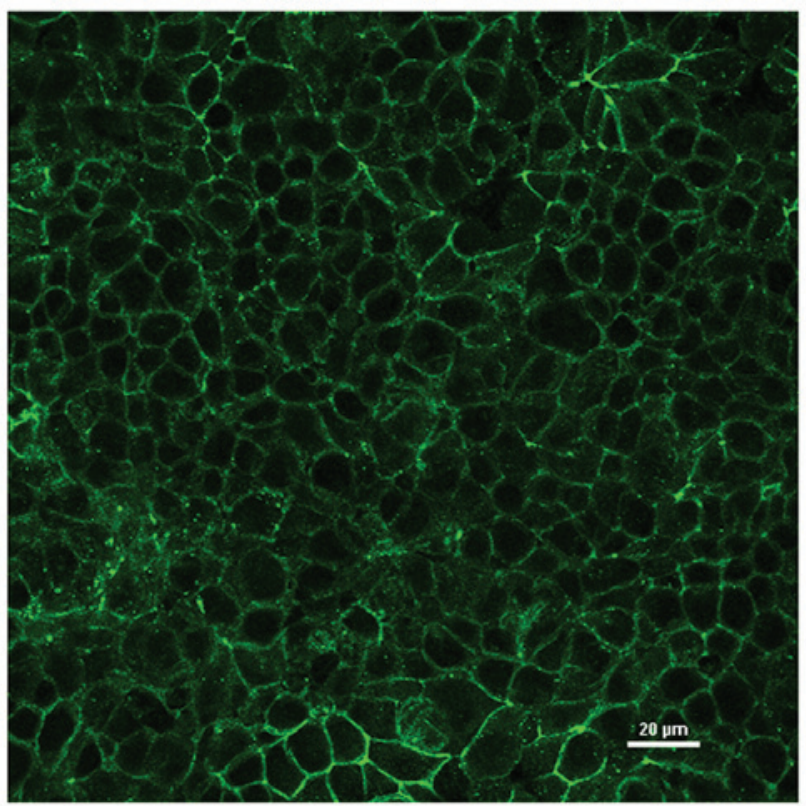

B

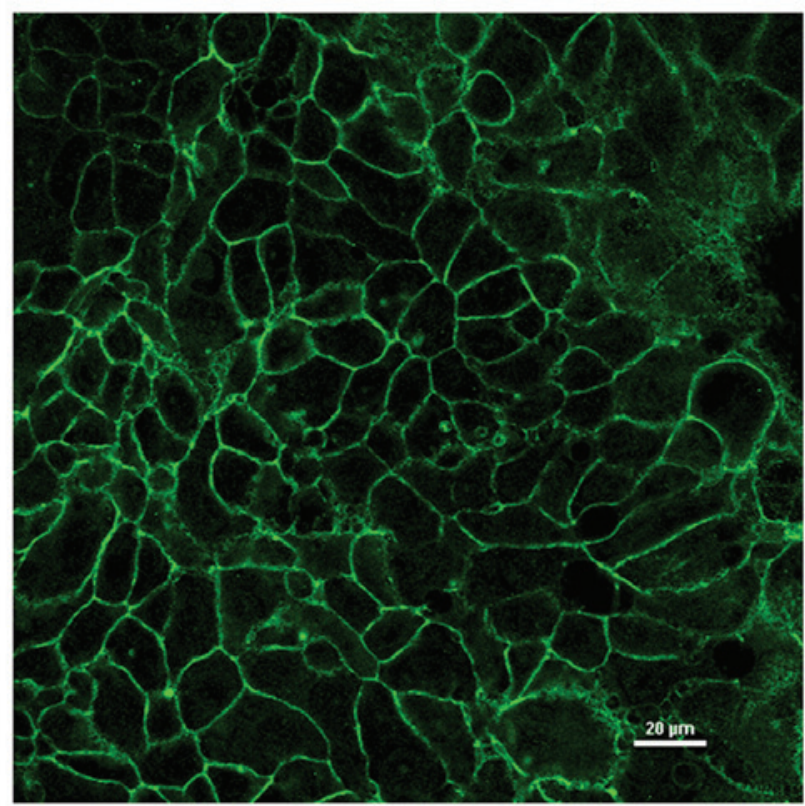

Figure 3. Subcellular localization of claudin-3 in breast cancer cell lines visualized by immunofluorescence. Breast cancer cells lines (A) MCF-7 and (B) MDA-MB-415 (overexpressing claudin-3) were plated onto chambered cover slides and incubated with an antibody to claudin-3. Claudin-3 protein was primarily observed at cell junctions but was also detected intracellularly (to a lesser extent) in both cell lines.
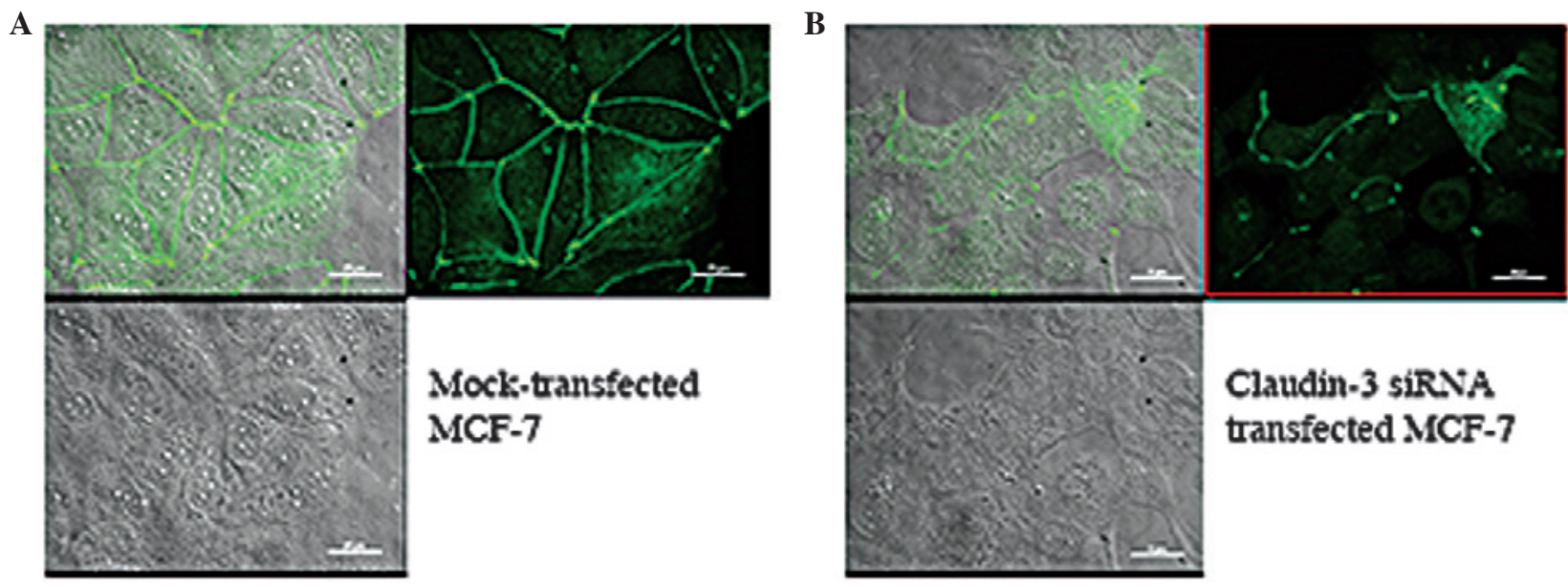

Figure 4. siRNA-mediated suppression of claudin-3 results in decreased junctional and intracellular fluorescence. (A) Mock-transfected and (B) claudin-3 siRNA-transfected MCF-7 cells were visualized by DIC microscopy (bottom panels) and claudin-3 localization was visualized by immunofluorescence (top right panels). The immunofluorescence images were overlaid onto the DIC images (top left panels). siRNA-transfected MCF-7 cells showed a decrease in claudin-3 signal intensity at the cell junctions and within the cell, relative to mock-transfected cells. DIC, differential interference contrast.

Cytoselect wound-healing assay was utilized to determine whether or not endogenous overexpression of claudin-3 protein is involved in promoting cellular motility of MCF-7 breast epithelial cells (Fig. 6). Whilst mock-transfected MCF-7 cells had fully migrated into the wound field by day 3 (Fig. 6A), a considerable lag was observed in the motility of the siRNA-transfected cells (Fig. 6B), associated with the sustained suppression of claudin-3 protein expression (Fig. 6C).

\section{Discussion}

Although much is known about the role of claudins in the regulation of paracellular transport across TJs, the extent of the functions of these proteins has yet to be fully elucidated.
In particular, the increasing evidence associating deregulation of claudins with tumorigenesis suggests that these proteins are important in multiple cellular processes, including motility and invasion (8-10), in addition to their established functions.

The present study compared the level of claudin-3 protein in a panel of different normal tissues to ascertain the variation in the basal levels of this protein. While the majority of the tissues expressed undetectable to low levels of claudin-3, normal breast tissue exhibited the highest, most readily detectable level of expression. The assessment of claudin-3 expression in three breast cancer cell lines revealed that, compared with normal breast epithelial cells (HMEC), there was a considerable overexpression of claudin-3 in two of the cell lines, MCF-7 and MDA-MB-415. By contrast, the third breast 
$\mathbf{A}$
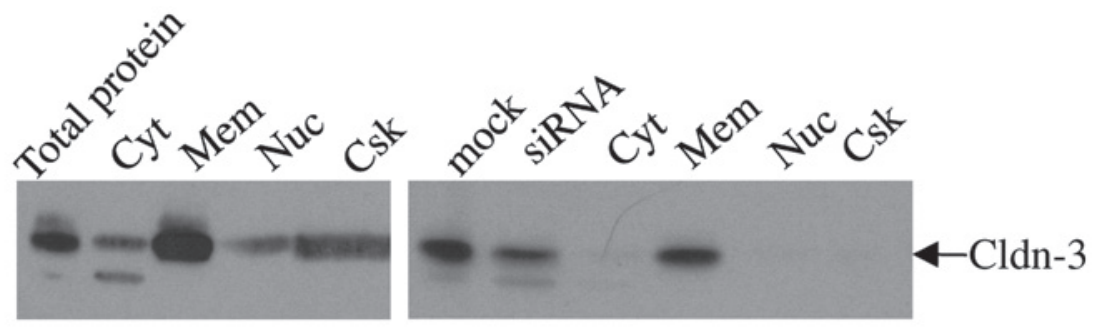

B
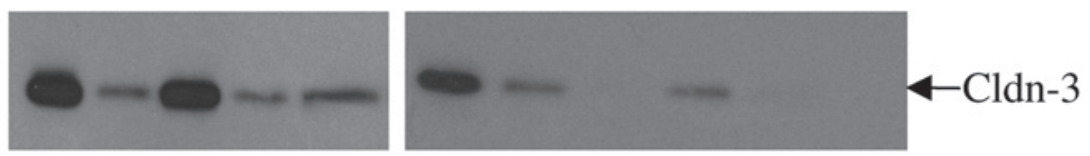

Figure 5. Subcellular localization of claudin-3 in mock versus siRNA-transfected MCF-7 and MDA-MB-415 by differential detergent fractionation of cells Subcellular fractions of mock or siRNA-transfected (A) MCF-7 and (B) MDA-MB-415 cells were immunoblotted with anti-claudin-3. The majority of claudin-3 was localized in the membrane fraction of non-transfected cells. Lower levels of claudin-3 were also observed in the cytosolic, nuclear and cytoskeletal fractions. siRNA-mediated suppression of claudin-3 resulted in an overall decrease in the level of claudin-3 protein in all sub-cellular fractions, with the greatest claudin-3 signal in the membrane fraction. Cyt, cytosolic; Mem, membranous; Nuc, nuclear; Csk, cytoskeletal; Cldn, claudin.

A

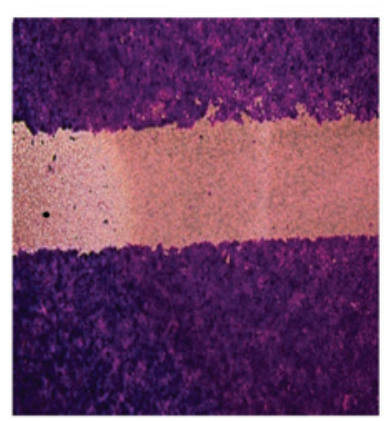

B

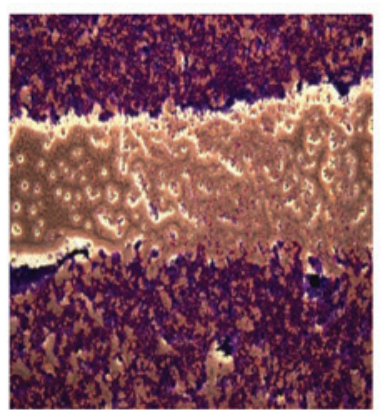

Day 0
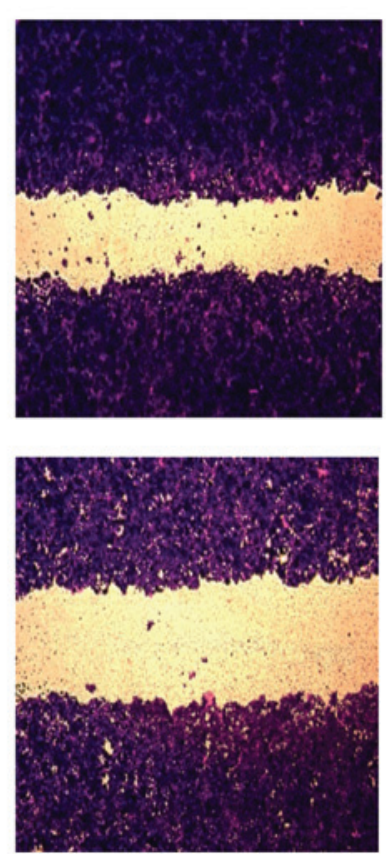

Day 1
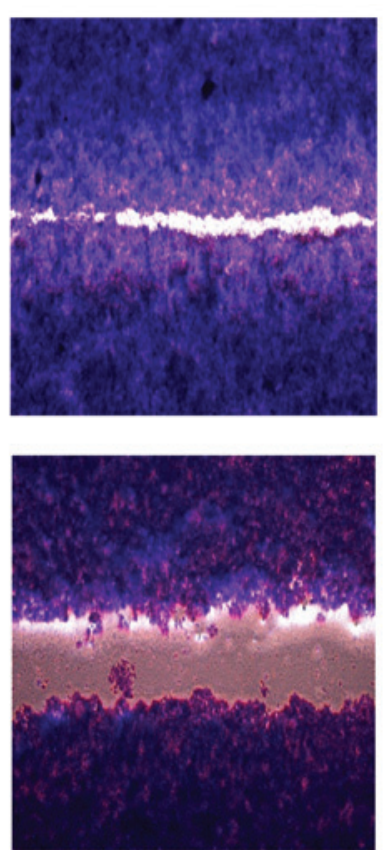

Day 2
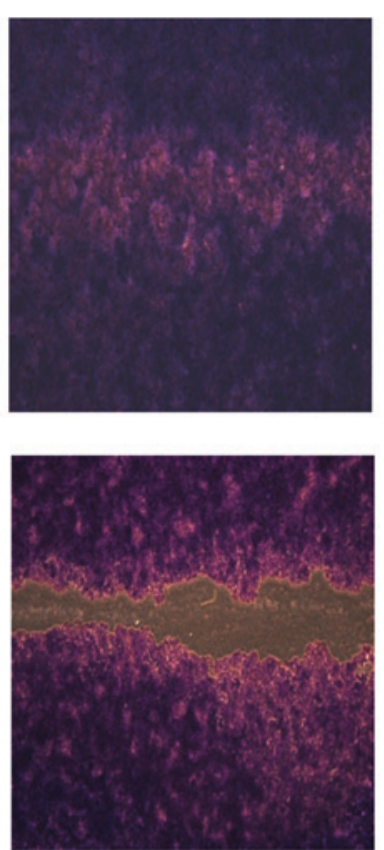

Day 3

C

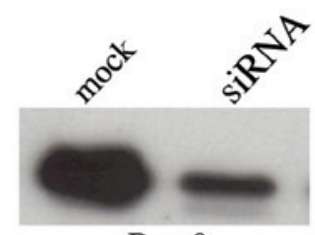

Day 0

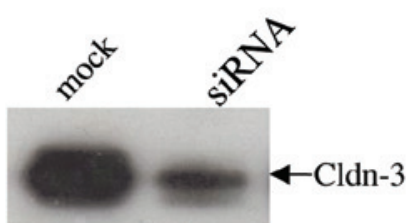

Day 3

Figure 6. siRNA-mediated suppression of claudin-3 decreases MCF-7 motility. Following transfection with claudin-3-specific siRNA, relative to that of (A) mock-transfected cells, the motility of (B) MCF-7 cells was markedly reduced. (C) Degree of suppression at the day 0 (two days post-siRNA transfection) and day 3 (five days post-siRNA transfection) time points. Cldn, claudin.

cancer cell line, MDA-MB-157, expressed a level of claudin-3 that was approximately equivalent to that of the HMEC cells. Notably, overexpression of claudin-4 protein was observed in the MCF-7 and MDA-MB-415 cell lines, in addition to a less striking overexpression of claudin-1 protein in all three breast cancer cell lines. The finding of abnormally elevated expression of claudin- 3 and -4 proteins in breast cancer cell lines is consistent with multiple studies that have demonstrated overexpression of claudins in a variety of tumor types. Specifically, elevated levels of claudin- 3 and -4 have been reported in ovarian $(8)$ and endometrial $(11,12)$ cancers, while claudin-4 overexpression has been reported in breast cancer (4). As previously stated, the MCF-7 and MDA-MB-415 cell lines are metastatic breast adenocarcinomas derived from pleural effusions. It is possible that the overexpression of claudin- 3 is involved in TJ disruption in these two breast tumors, thereby 
facilitating metastasis. Consistent with this, Agarwal et al (8) previously reported that exogenous overexpression of claudins-3 and -4 in ovarian cancer cell lines is associated with an increase in cell invasiveness and survival.

The present study also assessed the subcellular localization of claudin-3 protein in the MCF-7 and MDA-MB-415 breast cancer cell lines by immunofluorescence in order to determine whether or not abnormally elevated levels of the protein resulted in its delocalization. As predicted, both MCF-7 and MDA-MB-415 cells revealed intense membrane staining at cell junctions, consistent with the integral role of claudin-3 in TJs. Notably, intracellular fluorescence was also observed in both cell lines; this was, at least in part, attributable to the presence of claudin-3, as siRNA-mediated suppression of claudin-3 in MCF-7 cells also resulted in a decrease in intracellular fluorescence compared with mock-transfected cells. Consistent with the immunofluorescence data, differential detergent cell fractionation revealed low levels of claudin-3 in the cytoskeletal, nuclear and cytosolic fractions of mock- and siRNA-transfected MCF-7 and MDA-MB-415 cells, in addition to the predicted high level of claudin-3 in the membrane fractions. Notably, siRNA-mediated suppression of claudin-3 in MCF-7 and MDA-MB-415 cells resulted in an overall decrease in the level of claudin-3 protein in every cell fraction, however, the reduction appeared more prominent in the cytoskeletal fraction. The finding of intracellular claudin-3 in breast cancer cells expressing both elevated and suppressed levels of the protein supports the notion of additional roles for claudin proteins in cell signaling in tumor cells.

This abnormally elevated expression, coupled with the delocalization of claudin-3, suggests a possible function for $\mathrm{TJ}$ protein deregulation in local invasiveness of breast cancer cells. Therefore, the current study investigated the effect of claudin-3 overexpression on the motility of MCF-7 cells using a wound-healing assay. siRNA-mediated suppression of claudin-3 protein expression resulted in a decrease in the motility of MCF-7 cells. These data indicate that the deregulated expression of claudin-3 contributes to the invasive potential of breast cancer cells. Agarwal et al (8) previously reported enhanced cellular motility in ovarian epithelial cell lines engineered to stably overexpress claudin-3 and claudin-4 proteins. Furthermore, siRNA-mediated suppression of endogenously expressed claudin-3 and -4 in ovarian cancer cell lines resulted in a decrease in cellular motility (8). Similarly, Oku et al (9), showed a correlation between endogenous overexpression of claudin-1 protein and increased cellular invasion in oral squamous carcinoma. siRNA-mediated suppression of claudin-1 in these same cell lines also resulted in a decrease in invasiveness. Overexpression of claudin-1 has also been demonstrated in primary and metastatic colon tumors and cell lines (10). In the same study by Dhawan et al (10), suppression of claudin-1 expression in the metastatic colon cancer cell line, SW620, resulted in decreased motility, invasiveness and anchorage-independence (as assayed by soft agar). Consistent with the above studies, exogenous expression of claudin-1 in malignant melanoma cell lines results in delocalization of claudin-1 to the cytosol with increased cellular migration and elevated MMP-2 activity (13). By contrast, delocalization of exogenously expressed claudin-1 to the nucleus of the same malignant melanoma cell lines (often demonstrated by benign nevi) does not result in increased migration (13). These data suggest that the subcellular localization, in addition to the level of expression of claudins, may be important in the progression of tumors. Whereas the above reports suggest a role for overexpression of claudins in enhancing cellular motility, Michl et al (14) found that exogenous overexpression of claudin- 4 inhibited both the invasiveness and anchorage-independence of the pancreatic cell line, SUIT-2. Thus, it would appear that changes in claudin expression that deviate from the expression levels normally exhibited by normal cells of the same tissue type (including upregulation and downregulation of TJ proteins) contribute to the regulation of cellular motility and invasive potential.

Both the MCF-7 and MDA-MB-415 breast cancer cell lines used in the current study were derived from metastases in the lung, and thus it is possible that the overexpression and delocalization of claudin- 3 in these cells contributed to the progression of the original tumors. The use of siRNA to suppress claudin-3 expression may thus prove a beneficial gene therapeutic approach for cancers that exhibit deregulated expression and delocalization of claudins. If claudin-3 is involved in cell signaling, the preferential reduction of claudin-3 in the cytoskeletal fraction (observed in siRNA-transfected cells) may mediate the mechanism by which transduction of growth-stimulatory signals is attenuated in cancer cells.

\section{Acknowledgements}

This work was supported by the National Science Foundation Major Research Instrumentation Grant (grant no. 0922258), Joe and Jessie Crump Fund at JP Morgan Bank, the ACS Andrew W. Mellon Integrated Scholarly Grant and Howard Hughes Medical Institute through the Undergraduate Science Education Program (grant no. 52007558), and the Southwestern University Faculty-Student Collaborative Projects fund.

The authors would also like to thank Ms. Bronwyn Tyler for her technical assistance and Mr. Aidan Todd for his assistance in the wound-healing assays.

\section{References}

1. American Cancer Society: Breast Cancer Facts \& Figures http://www.cancer.org/research/cancerfactsstatistics/ breast-cancer-facts-figures. Accessed February 24, 2014.

2. Turksen K and Troy TC: Barriers built on claudins. J Cell Sci 117: 2435-2447, 2004

3. Kominsky SL, Vali M, Korz D, Gabig TG, Weitzman SA Argani P and Sukumar S: Clostridium perfringens enterotoxin elicits rapid and specific cytolysis of breast carcinoma cells mediated through tight junction proteins claudin 3 and 4 . Am J Pathol 164: 1627-1633, 2004.

4. Lanigan F, McKiernan E, Brennan DJ, Hegarty S, Millikan RC, McBryan J, et al: Increased claudin-4 expression is associated with poor prognosis and high tumour grade in breast cancer. Int J Cancer 124: 2088-2097, 2009.

5. Tokés AM, Kulka J, Paku S, Szik A, Páska C, Novák PK, Szilák L, Kiss A, Bögi K and Schaff Z: Claudin- $1,-3$ and -4 proteins and mRNA expression in benign and malignant breast lesions: a research study. Breast Cancer Res 7: R296-R305, 2005.

6. Kominsky SL, Argani P, Korz D, Evron E, Raman V, Garrett E, Rein A, Sauter G, Kallioniemi OP and Sukumar S: Loss of the tight junction protein claudin-7 correlates with histological grade in both ductal and carcinoma in situ and invasive ductal carcinoma of the breast. Oncogene 22: 2021-2033, 2003. 
7. Tokés AM, Kulka J, Paku S, Máthé M, Páska C, Lódi C. Kiss A and Schaff Z: The expression of five different claudins in invasive breast carcinomas: comparison of pT1pN1 and pT1pN0 tumors. Pathol Res Pract 201: 537-544, 2005.

8. Agarwal R, D'Souza T and Morin PJ: Claudin-3 and claudin-4 expression in ovarian epithelial cells enhances invasion and is associated with increased matrix metalloproteinase-2 activity. Cancer Res 65: 7378-7385, 2005.

9. Oku N, Sasabe E, Ueta E, Yamamoto T and Osaki T: Tight junction protein claudin-1 enhances the invasive activity of oral squamous cell carcinoma cells by promoting cleavage of laminin-5 gamma2 chain via matrix metalloproteinase (MMP)-2 and membrane-type MMP-1. Cancer Res 66: 5251-5257, 2006.

10. Dhawan P, Singh AB, Deane NG, No Y, Shiou SR, Schmidt C, Neff J, Washington MK and Beauchamp RD: Claudin-1 regulates cellular transformation and metastatic behavior in colon cancer. J Clin Invest 115: 1765-1776, 2005.

11. Pan XY, Wang B, Che YC, Weng ZP, Dai HY and Peng W: Expression of claudin-3 and claudin-4 in normal, hyperplastic, and malignant endometrial tissue. Int J Gynecol Cancer 17: 233-241, 2007
12. Konecny GE, Agarwal R, Keeney GA, Winterhoff B, Jones MB, Mariani A, et al: Claudin-3 and claudin-4 expression in serous papillary, clear-cell, and endometroid endometrial cancer. Gynecol Oncol 109: 263-269, 2008.

13. French AD, Fiori JL, Camilli TC, Leotlela PD, O'Connell MP, Frank BP, Subaran S, Indig FE, Taub DD and Weeraratna AT: PKC and PKA phosphorylation affect the subcellular localization of claudin-1 in melanoma cells. Int J Med Sci 6: 93-101, 2009.

14. Michl P, Barth C, Buchholz M, Lerch MM, Rolke M, Holzmann KH, et al: Claudin-4 expression decreases invasiveness and metastatic potential of pancreatic cancer. Cancer Res 63: 6265-6271, 2003. 